\title{
Maximum Likelihood Estimation of Time-series with Markov Regime
}

\author{
Subhrakanti Dey, student member, IEEE, Vikram Krishnamurthy, member, IEEE \\ Department of Systems Engineering, \\ Research School of Information Sciences and Engineering, \\ Australian National University, Canberra ACT 0200, Australia \\ Tel: +61 62493259 Fax: +616279 8088 E-mail: subhra@syseng.anu.edu.au \\ Thierry Salmon-Legagneur \\ DSTO, Australia
}

\begin{abstract}
In this paper, we consider the estimation of various Markov-modulated time-series. We obtain maximum likelihood estimates of the time-series parameters including the Markov chain transition probabilities and the timeseries coefficients using the EM (Expectation Maximization) algorithm. Also the recursive EM algorithm is used to obtain on-line parameter estimates. Simulation studies show that both algorithms yield satisfactory results.
\end{abstract}

\section{Introduction}

Signal Model: Let $s_{k}$ denote a $N_{s}$-state irreducible Markov chain with states $\left\{1,2, \ldots, N_{,}\right\}$with transition probability matrix $\Pi=\left(\pi_{m n}\right), \pi_{m n}=P\left(s_{k+1}=n \mid s_{k}=\right.$ $m)$ and initial state probability $\pi=\left(\pi_{m}\right), \pi_{m}=P\left(s_{1}=\right.$ $m$ ). Define the Markov-modulated polynomials as follows:

$$
\begin{aligned}
& A\left(z^{-1}, s_{k}\right)=1+\sum_{i=1}^{p} a_{i}\left(s_{k}\right) z^{-i} \\
& B\left(z^{-1}, s_{k}\right)=1+\sum_{i=1}^{q} b_{i}\left(s_{k}\right) z^{-i} \\
& C\left(z^{-1}, s_{k}\right)=1+\sum_{i=1}^{r} c_{i}\left(s_{k}\right) z^{-i}
\end{aligned}
$$

where $z^{-1}$ denotes the delay operator and $k$ denotes discrete-time. Let $A(m) \triangleq\left(a_{1}(m) \ldots a_{p}(m)\right)^{\prime}, B(m) \triangleq$ $\left(b_{1}(m) \ldots b_{q}(m)\right)^{\prime}, C(m) \triangleq\left(c_{1}(m) \ldots c_{r}(m)\right)^{\prime}$. In this paper, we consider estimation of any one of the following second-order stationary Markov-modulated timeseries models:

$$
\begin{array}{rc}
\text { ARX: } & A\left(z^{-1}, s_{k}\right) y_{k}=B\left(z^{-1}, s_{k}\right) u_{k}+w_{k} \\
\text { MAX : } & y_{k}=B\left(z^{-1}, s_{k}\right) u_{k}+C\left(z^{-1}, s_{k}\right) w_{k} \\
\text { ARMA : } & A\left(z^{-1}\right) y_{k}=C\left(z^{-1}, s_{k}\right) w_{k}
\end{array}
$$

where $u_{k}, y_{k}$ are the measured input and output at time $k, w_{k} \sim$ white $N\left(0, \sigma^{2}\right)$ is independent of $s_{k}$ and $\phi$ is the parameter vector consisting of polynomial coefficients and Markov chain parameters (e.g., $\phi=\left(A(m), B(m), \Pi, \sigma^{2}\right)$ for (2)). We assume $u_{k}$ to be persistently exciting [4]. We also assume that $A\left(z^{-1}, s_{k}\right), B\left(z^{-1}, s_{k}\right)$ and $C\left(z^{-1}, s_{k}\right)$ are coprime to each other for each $m, m \in\left\{1,2, \ldots, N_{s}\right\}$.
Notations: $Y_{k}=\left(y_{1}, \ldots, y_{k}\right)^{T}, U_{k}=\left(u_{1} \ldots u_{k}\right)^{T}, Z_{k}=$ $\left(Y_{k}, U_{k}\right)$ denotes the observed "incomplete" data. $S_{k}=$ $\left(s_{1} \ldots s_{k}\right)^{T}, Y_{t}^{k}=\left(y_{t} \ldots y_{k}\right)^{T}$ and $U_{t}^{k}=\left(u_{t} \ldots u_{k}\right)^{T}$ where superscript $T$ denotes transpose.

Estimation Objectives: We use the Expectation Maximization (EM) algorithm [7] to obtain maximum likelihood (ML) estimates of $\phi$, given $Y_{T}, U_{T}$ (when appropriate) in Sec. 2. Also based on the recursive EM algorithm [2], an on-line estimation scheme is presented in Sec. 3.

In [5], the EM algorithm and a recursive EM algorithm are used to estimate Markov-modulated AR processes which is a special case of our model (2) with $B=0$. The three models we consider in this paper can be regarded as an extension of the work in [5]. Applications of such estimation algorithms can be found in [6], [5] and in the references therein.

Remark 1: Models (2), (3) or (4) are special cases of the Markov-modulated ARMAX model

$$
A\left(z^{-1}, s_{k}\right) y_{k}=B\left(z^{-1}, s_{k}\right) u_{k}+C\left(z^{-1}, s_{k}\right) w_{k}
$$

However, unlike (2), (3) and (4), ML estimation of (5) is computationally prohibitive since it requires computing probability density functions over all $N_{0}^{T}$ realisations of a $N$, state $T$ point Markov chain. For similar reasons, we forbid $A\left(z^{-1}\right)$ in (4) to be Markov-modulated.

Remark 2: Deriving stationarity criteria for Markovmodulated time-series is a difficult problem. For example, two switching, separately second order AR stationary processes can result in an unstable system - whereas two individually unstable $A R$ processes can be stabilized when allowed to switch according to a Markov regime. For sufficient conditions on the second-order stationarity of Markov-modulated time- series, see [5].

\section{ML estimation via EM algorithm}

Markov-modulated ARX estimation The EM algorithm is an iterative procedure; each iteration involves two steps, E-step and M-step.

E Step: Following [3], the expectation of the log-likelihood function of a $T$-point "complete" data sequence $M_{T}=$ $\left(Y_{T}, U_{T}, S_{T}\right)$ defined as

$$
\mathcal{Q}\left(\phi^{(l)}, \phi\right) \triangleq E\left\{\ln f\left(M_{T} \mid \phi\right) \mid Z_{T}, \phi^{(1)}\right\}
$$




$$
\begin{aligned}
= & -\frac{T}{2} \ln \sigma^{2}-\frac{1}{2 \sigma^{2}} \sum_{k=1}^{T-1} \sum_{m=1}^{N_{\infty}} \gamma_{k}(m)\left(A\left(z^{-1}, m\right) y_{k}-\right. \\
& \left.B\left(z^{-1}, m\right) u_{k}\right)^{2}+\sum_{k=1}^{T-1} \sum_{m=1}^{N_{s}} \sum_{n=1}^{N_{s}} \xi_{k}(m, n) \ln \pi_{m n} \\
& +\sum_{m=1}^{N_{S}} \gamma_{1}(m) \ln \pi_{m}
\end{aligned}
$$

where $\xi_{k}(m, n) \triangleq f\left(s_{k}=m, s_{k+1}=n \mid Z_{T}, \phi^{(l)}\right)$ and $\gamma_{k}(m) \triangleq f\left(s_{k}=m \mid Z_{T}, \phi^{(l)}\right) . \gamma_{k}(m)$ is computed via the "forward backward" procedure described in [1]. $\phi^{(l)}$ is the estimate of the parameter vector at the $l$-th iteration assuming the iteration procedure starts with an initial estimate $\phi^{(0)}$.

M Step: This step involves computing arg $\max _{\phi} Q\left(\phi^{(l)}, \phi\right)$ to yield the estimates of $\pi_{m n}, \sigma^{2}, A(m), B(m)$. For all the relevant details, see [6].

Markov-modulated MAX estimation

The MAX model (3) can be written in equivalent ARX form as

$$
A^{\prime}\left(z^{-1}, s_{k}\right) y_{k}=B^{\prime}\left(z^{-1}, s_{k}\right) u_{k}+e_{k}
$$

where the polynomial $A^{\prime}\left(z^{-1}, s_{k}\right)$ is "sufficiently" long enough to ensure that $e_{k}$ is almost white (see [4], pg 291 for details) and $B^{\prime}\left(z^{-1}, s_{k}\right)=A^{\prime}\left(z^{-1}, s_{k}\right) B\left(z^{-1}, s_{k}\right)$. The EM algorithm described in the previous section yields the estimates of $A^{\prime}(m)$ and $B^{\prime}(m)$ and hence of $B(m) . C(m)$ in (3) can be estimated by solving a set of inverse YuleWalker equations (see pg 291, [4]). Details can be found in [6].

Markov-modulated ARMA estimation

Since $A$ in (4) is no longer Markov-modulated, it can be estimated via a set of Yule-Walker equations (see pp 289, [4]). Rewriting (4) as

$$
A\left(z^{-1}\right) A^{\prime}\left(z^{-1}, s_{k}\right) y_{k}=e_{k}
$$

(where $e_{k}$ and $A^{\prime}\left(z^{-1}, s_{k}\right)$ are as defined in the previous section), estimate of $A\left(z^{-1}\right) A^{\prime}\left(z^{-1}, s_{k}\right)$ and hence $C(m)$ can be obtained via EM.

\section{On-line Estimation via Recursive EM algorithm}

An on-line estimation scheme can be implemented based on the recursive EM algorithm proposed in [2].

\section{Simulation studies}

We present simulation examples, with $N_{s}=2, \pi_{11}=$ $\pi_{22}=0.9$ for on-line recursive EM algorithm. Simulation results for the off-line EM algorithm can be found in [6]. On-line estimation via recursive EM algorithm Consider a jump time-varying 100000 point
Markov-modulated MAX model with $\sigma^{2}=1$ and

$$
\begin{aligned}
& B(1)=\left(\begin{array}{lll}
0.8 & 0.3
\end{array}\right)^{\prime}, B(2)=\left(\begin{array}{lll}
0.5 & 0.1
\end{array}\right)^{\prime}, C(1)=\left(\begin{array}{ll}
0.5 & 0.3
\end{array}\right)^{\prime}, \\
& C(2)=(-0.40 .2)^{\prime} \quad t \leq 20000
\end{aligned}
$$

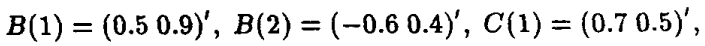

$$
\begin{aligned}
& C(2)=(-0.20 .5)^{\prime} \quad t>20000
\end{aligned}
$$

Figure 1 shows the time evolution of the estimates when the estimation procedure starts with arbitrary initial estimates. Results for a Markov-modulated ARMA model can be found in [6].
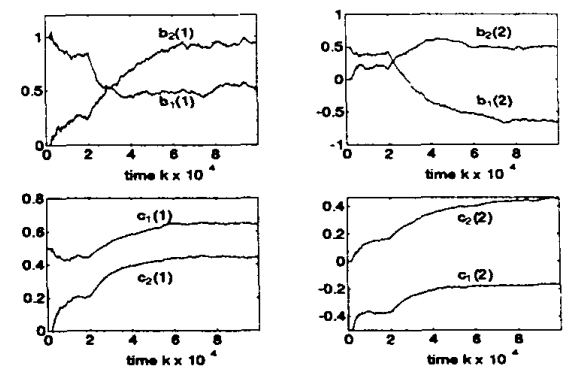

Figure 1: Time evolution of MAX parameters

\section{References}

[1] L.R. Rabiner, "A tutorial on Hidden Markov Models and selected applications in speech recognition," Proc. IEEE, Vol.77, No.2, pp 257-285, 1989.

[2] V. Krishnamurthy, J.B. Moore, "On-line Estimation of Hidden Ma rkov Model Parameters based on the Kullback-Leibler Information Measure," IEEE Trans. on Signal Processing, Vol. 41, No. 8, pp. 25572573, August, 1993.

[3] D.M. Titterington, A.F.M. Smith and U.E. Makov, Statistical Analysis of Finite Mixture Distributions, New York, Wiley, 1985.

[4] T. Söderström, P. Stoica, System Identification, Prentice Hall, 1989.

[5] U. Holst, G. Lindgren, J. Holst and M. Thuvesholmen, "Recursive Estimation in Switching Autoregressions with Markov Regime," to appear in Journal of Time Series Analysis, 1994.

[6] S. Dey, V. Krishnamurthy and T. Salmon-Legagneur, "Estimation of Markov-modulated Time-series via EM algorithm," to appear in IEEE Signal Processing Letters, October 1994.

[7] A.P. Dempster, N.M Laird, D.B. Rubin, "Maximum likelihood from incomplete data via the EM algorithm," J. Royal Stat. Soc., ser 39, vol. 6, pp 1-38, 1977. 